\title{
Enhancement of Macromolecular Ice Recrystallization Inhibition Activity by Exploiting Depletion Forces
}

\author{
Toru Ishibe, $^{\dagger}$ Thomas Congdon, ${ }^{\dagger}$ Christopher Stubbs, ${ }^{\dagger}$ Muhammad Hasan, ${ }^{\dagger}$ Gabriele C. Sosso, ${ }^{\dagger} \S_{\odot}$
} and Matthew I. Gibson*, $*$,
${ }^{\dagger}$ Department of Chemistry, ${ }^{\ddagger}$ Warwick Medical School, and ${ }^{\S}$ Centre for Scientific Computing, University of Warwick Coventry, CV4 7AL, United Kingdom

Supporting Information

\begin{abstract}
Antifreeze (glyco) proteins (AF(G)Ps) are potent inhibitors of ice recrystallization and may have biotechnological applications. The most potent $\mathrm{AF}(\mathrm{G}) \mathrm{Ps}$ function at concentrations a thousand times lower than synthetic mimics such as poly(vinyl alcohol), PVA. Here, we demonstrate that PVA's ice recrystallization activity can be rescued at concentrations where it does not normally function, by the addition of noninteracting polymeric depletants, due to PVA forming colloids in the concentrated saline environment present between ice crystals. These depletants shift the equilibrium toward ice binding and, hence, enable PVA to inhibit ice growth at lower concentrations. Using theory and experiments, we show this effect requires polymeric depletants, not small molecules, to enhance activity. These results increase our understanding of how to design new ice growth inhibitors, but also offer opportunities to enhance activity by exploiting depletion forces, without re-engineering icebinding materials. It also shows that when screening for IRI activity that polymer contaminants in buffers may give rise to false positive results.
\end{abstract}

\begin{abstract}
A ntifreeze proteins are ice binding proteins that control ice crystal growth and are found in a variety of extremophile organisms. ${ }^{1}$ Their ability to modulate ice growth means they have generated interest in application areas where ice growth is a problem, especially in the cryopreservation of donor cells and tissues. Several classes of synthetic mimics ${ }^{2-5}$ of antifreeze proteins have been developed, including glycopeptides, ${ }^{6,7}$ poly(ampholytes), ${ }^{8-10}$ and amphipathic molecules/assemblies. ${ }^{11-14}$ Poly(vinyl alcohol) has emerged as a particularly active mimic, shows molecular weight dependent activity, ${ }^{15-18}$ and may have application in cryopreservation. ${ }^{19,20}$ Molecular modeling suggests that PVA's activity is due to the precise spacing of its hydroxyl groups, which match the spacing in the prismatic plane of ice, and this effect increases with molecular weight due to entropy/enthalpy compensation. ${ }^{21,22}$ There have been investigations into whether antifreeze proteins bind to ice reversibly or irreversibly, to account for their concentration dependence. Microfluidic experiments have, however, provided evidence that AFPIII and AFGP bind irreversibly, ${ }^{23,24}$ but PVA seems to be a reversible binder and contributes to its lower activity. If PVA is binding reversibly, this means an equilibrium is established between ice-bound and unbound PVA, with only the bound PVA molecules contributing to the observable macroscopic properties. Hence, a large fraction of the added macromolecules does not contribute to activity, but is required to drive the equilibrium toward ice binding. In colloid science,
\end{abstract}

it is well-known that the addition of noninteracting macromolecules to a colloidal dispersion can cause aggregation and shift them from dispersion to a surface. This is due to the entropically driven depletion force in which polymeric depletants are excluded from the volume between particles, leading to aggregation. This process underpins structural biology, where polymeric depletants (especially poly(ethylene glycol)) are used in protein crystallization buffers to enable structural studies. ${ }^{25}$ Castro et al. developed DNA origami devices that refold in response to small depletion forces. ${ }^{26}$ Chen and co-workers showed how depletion effects could be used to tune the morphology and self-assembly of block copolymers in the presence of a nonabsorbing homopolymer. ${ }^{27}$ Nagao and co-workers utilized depletants to drive the selfassembly of golf ball-like particles to form branched chains. ${ }^{28}$ Poly(ethylene glycol) (PEG) has been shown to act as a depletant for colloids. ${ }^{29}$ Kuhl et al. were able to directly measure the depletion force induced by PEG on egg phosphatidylcholine vesicles, showing the molecular weight dependence. $^{30}$ The depletion effect, also known as macromolecular crowding, is partly responsible for protein

Received: May 22, 2019

Accepted: August 7, 2019

Published: August 9, 2019 
folding, ${ }^{31,32}$ the assembly of the cytoskeleton, and whole chromosomes. $^{33}$

Considering the above, we reasoned that, if the PVA was forming colloids, then addition of a secondary polymer, which has no IRI activity, could act as a depletant to drive the PVA to the ice and, hence, shift the equilibrium to favor ice binding, enabling activity at lower concentrations.

Here we report that the addition of secondary polymers which do not target any ice crystal faces can enhance the IRI activity of poly(vinyl alcohol) by forcing the equilibrium toward binding due to depletion forces. The enhancement is shown to require a polymer, not small molecules, and is universally applicable.

Figure 1A illustrates the calculated depletion force for PVA aggregation in the presence of PEG. When the saline $([\mathrm{NaCl}]$
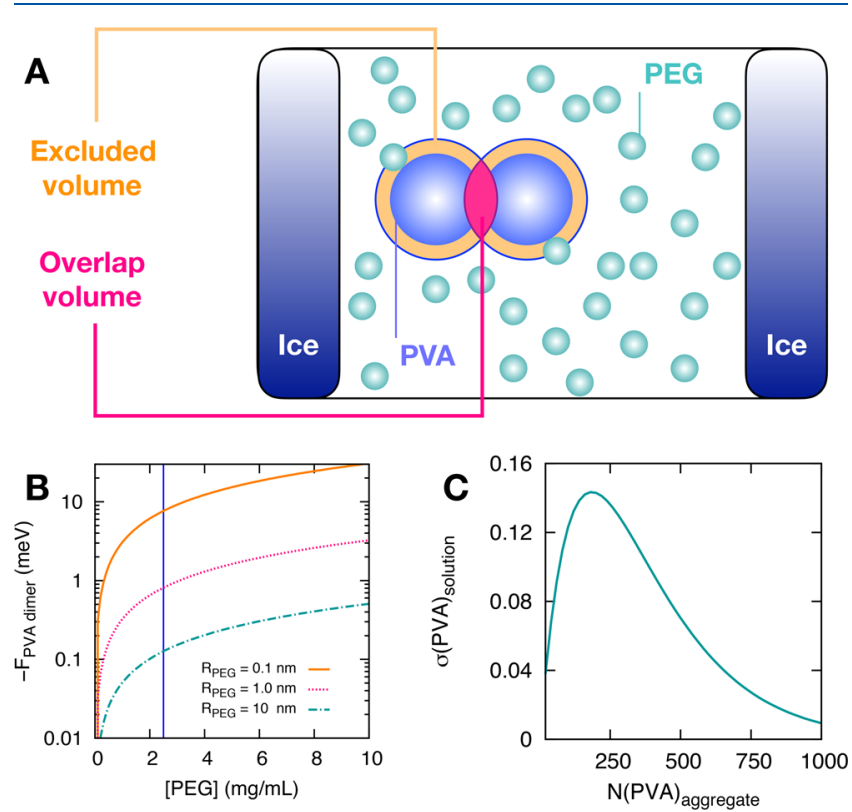

Figure 1. Depletion effects as the driving force for PVA aggregation in the presence of PEG particles. (A) Schematics of the origin of depletion forces: as the PEG particles cannot overlap with the PVA particles, the former cannot access a certain excluded volume around the latter. However, the excluded volume is reduced by the overlap volume created when two PVA particles get close enough. Thus, the aggregation of PVA increases the volume available to the PEG, which in turn leads to an entropic gain for the system. (B) Calculated free energy gain due to the aggregation of two PVA particles into a "dimer", as a function of PEG concentration. (C) Calculated volume fraction $\sigma(\mathrm{PVA})_{\text {solution }}$ of PVA particles participating in PVA aggregates of size N(PVA) aggregate. The details of all these calculations are reported in the ESI.
$=0.14 \mathrm{M}$ ) solutions used to assess IRI activity freeze, ice crystals exclude other solutes and hence concentrate them between ice grains to give unfrozen water channels. ${ }^{34}$ As seen using DLS (dynamic light scattering), PVA aggregates under these conditions (see ESI), but PEG (which has no IRI activity) does not. As depicted in Figure 1A, the aggregation of PVA particles reduces the excluded volume of the system which is not accessible to PEG (modeled as a small particle). This leads in turn to a net entropic gain for the system. The Asakura-Oosawa model for depletion forces was used to estimate the free energy gain resulting from the aggregation of (radius $=10 \mathrm{~nm}$ ) PVA particles in the presence of (nonaggregated, radius $=1 \mathrm{~nm}$ ) PEG particles. Full details of the model can be found in the Supporting Information. As shown in Figure 1B, the free energy gain due to depletion forces is strongly dependent on the size of the depletant, which has been observed experimentally by others using a PEG system. ${ }^{29,30}$ Smaller PEG (but still a polymer) leads to much stronger PVA-PVA interactions, where a larger (radius 10 $\mathrm{nm}$ ) depletant has less effect, showing a macromolecular, but not-particulate, depletant is required (confirmed experimentally). In Figure $1 \mathrm{C}$ we report the volume fraction of PVA particles participating in PVA aggregates containing $\mathrm{N}$ (PVA) aggregate particles; Here, we have assumed a PVA concentration of $2.5 \mathrm{mg} \cdot \mathrm{mL}^{-1}$ and a volume fraction of PEG of 0.38 (assuming a 100-fold increase of both PVA and PEG concentration upon confinement between ice crystals during the experiments). In this case, most of the PVA can only be found as aggregates of substantial size ( $\sim 250$ polymers). While estimates, these results provide a simple motivation for the aggregation of PVA particles due to the depleting action of PEG.

To enable experimental investigation of this depletion effect, a range of polymers were synthesized by RAFT (reversible additional fragmentation chain transfer) polymerization to be tested as depletants along with commercial PEG. Full details of the synthesis are in the Supporting Information, and the polymers are shown in Table 1 .

PVA with DP $=85\left(\mathrm{PVA}_{85}\right)$ was selected to ensure the polymer was well above the critical minimum length required for ice growth inhibition. ${ }^{16,17,22}$ The "splat" assay was used to evaluate its concentration-dependent IRI activity; This involves seeding a polynucleated ice wafer, which is annealed at $-8{ }^{\circ} \mathrm{C}$ for $30 \mathrm{~min}$, and the average crystal size compared against a negative control. The mean grain size (MGS) is reported and smaller numbers indicate greater IRI activity. A concentration of $\mathrm{PVA}_{85}$ of $0.025 \mathrm{mg} \cdot \mathrm{mL}^{-1}$ gave $50 \%$ MGS and was used from this point on to ensure that any changes in activity can be easily observed. Figure $2 \mathrm{~A}$ shows the MGS of $\mathrm{PVA}_{85}$ supplemented with/out PEG4k. PEG4k alone shows no IRI

\section{Table 1. Polymers Used in This Study}

\begin{tabular}{|c|c|c|c|c|c|c|}
\hline $\operatorname{code}^{a}$ & {$[\mathrm{M}]:[\mathrm{CTA}]^{b}(-)$} & $\operatorname{conv}^{c}(\%)$ & $\mathrm{Mn}_{\text {Theo }}{ }^{d}\left(\mathrm{~g} \cdot \mathrm{mol}^{-1}\right)$ & $\mathrm{Mn}_{\mathrm{NMR}}{ }^{e}\left(\mathrm{~g} \cdot \mathrm{mol}^{-1}\right)$ & $\mathrm{Mn}_{\mathrm{SEC}}\left(\mathrm{g} \cdot \mathrm{mol}^{-1}\right)$ & $\bigoplus^{f}$ \\
\hline $\mathrm{PVAc}_{85}$ & 100 & 90.0 & 7700 & 8200 & 7300 & 1.50 \\
\hline PAA & 100 & 94.2 & 6800 & 12000 & $1040^{g}$ & $\mathrm{~N} / \mathrm{A}^{g}$ \\
\hline PMA & 100 & 86.6 & 7500 & $7700^{h}$ & 14176 & 2.72 \\
\hline $\mathrm{PVP}_{12}$ & 20 & 74.3 & 1700 & 1300 & 1300 & 1.40 \\
\hline $\mathrm{PVP}_{206}$ & 300 & 65.4 & 22000 & 15000 & 23000 & 1.51 \\
\hline
\end{tabular}

${ }^{a}$ Polymers are named according to the DP from SEC. ${ }^{b}$ Monomer to CTA ratio. ${ }^{c}$ Determined by NMR compared to an internal standard. ${ }^{d}$ Determined by target MW multiplied by conversion. ${ }^{e}$ Determined by end group analysis. ${ }^{f}$ Determined by SEC. ${ }^{g}$ Significant tailing was observed in the aqueous GPC, leading to high dispersity values $(>4) .{ }^{h}$ Determined from NMR conversion as end groups not visible. 


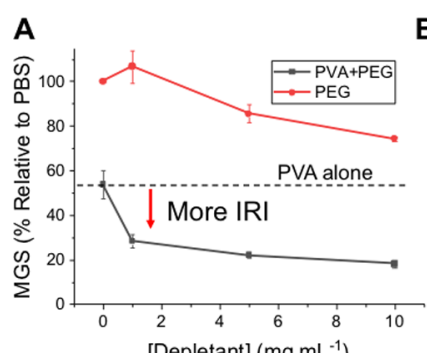

B
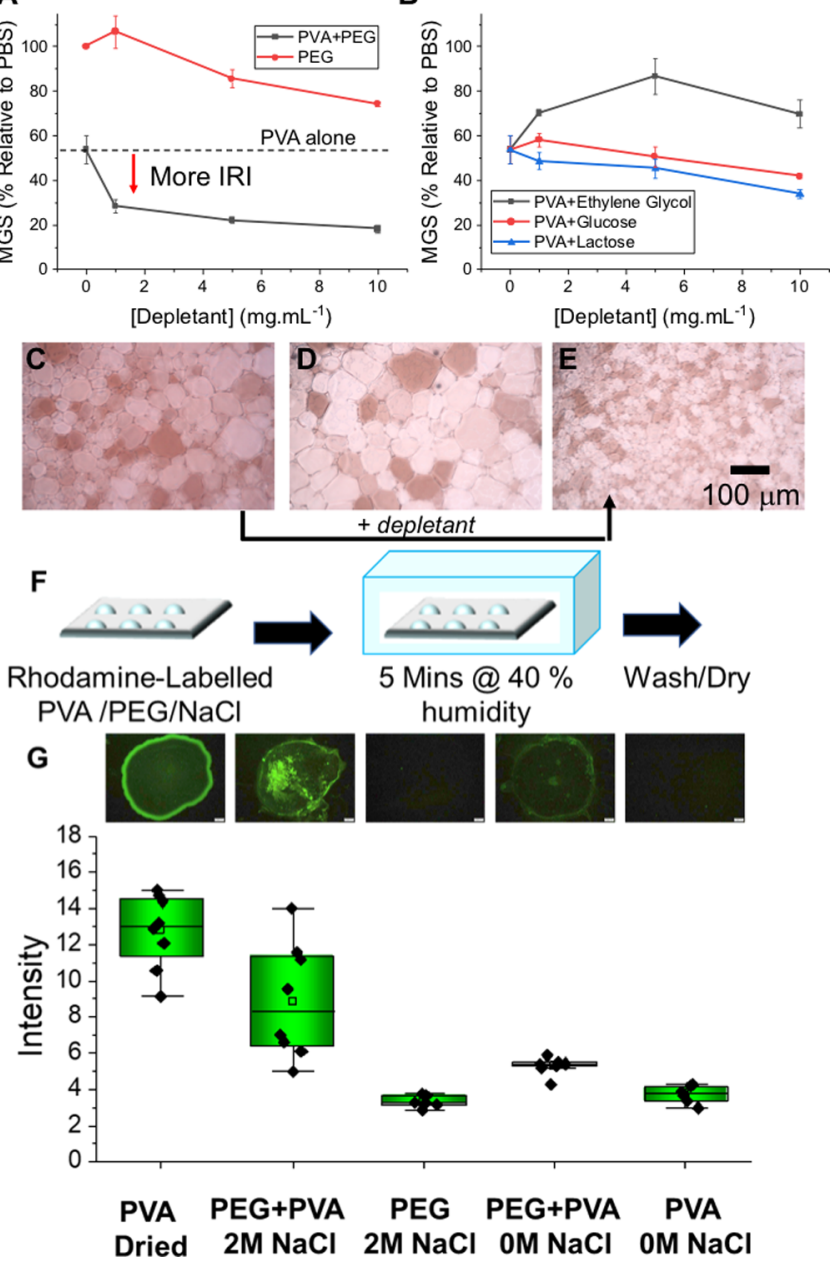

Figure 2. Effect of PEG as a depletant to modulate IRI activity. (A) IRI activity of $\mathrm{PVA}_{85}\left(0.025 \mathrm{mg} \cdot \mathrm{mL}^{-1}\right)$ with PEG 4k; (B) PVA 85 IRI activity with small molecule additives; Example cryomicroscopy images for (C) $\mathrm{PVA}_{85}\left(0.025 \mathrm{mg} \cdot \mathrm{mL}^{-1}\right)$, (D) PEG $4 \mathrm{k}\left(10 \mathrm{mg} \cdot \mathrm{mL}^{-1}\right)$, (E) PVA $_{85}\left(0.025 \mathrm{mg} \cdot \mathrm{mL}^{-1}\right)+$ PEG $4 \mathrm{k}\left(10 \mathrm{mg} \cdot \mathrm{mL}^{-1}\right)$, (F) Schematic of depletion testing onto glass slides, and (G) Fluorescence microscopy and image analysis of Rhodamine-labeled PVA being depleted by PEG and $\mathrm{NaCl}$. Average intensity values from minimum of 6 droplets. Scale bar $=100 \mu \mathrm{m}$. MGS $=$ mean grain size.

activity, confirming it has no direct ice interaction. However, addition of PEG4k to $\mathrm{PVA}_{85}$ enabled the rescue of activity, with $10 \mathrm{mg} \cdot \mathrm{mL}^{-1}$ rescuing all activity with a MGS of $20 \%$. The observed activity profile is in close agreement with the theoretical calculations (Figure 1). Theory predicts that only polymers can act as depletants, which was confirmed by the lack of enhancement when mono/disaccharides and ethylene glycol are added. To visualize the depletion effect, Rhodaminelabeled PVA was incubated with PEG, with and without $\mathrm{NaCl}$ (to mimic the conditions between ice wafers) on a glass slide, Figure $2 \mathrm{~F}$. In the presence of a depletant, the polymer will be forced onto the interface between the glass and the droplet, adhering to the surface. Image analysis (Figure $2 \mathrm{G}$ ) confirmed that PVA was depleted and forced to the glass surface only when PEG and $\mathrm{NaCl}$ were present, in agreement with the ice growth data. It can be seen in Figure $2 \mathrm{G}$ that when fluorescent PVA is dried on the glass surface, the majority of the polymer accumulates at the air/water/glass interface.
If the depletion hypothesis is true, it should also be valid for other polymers. Figure 3 shows the observed MGS for $\mathrm{PVA}_{85}$

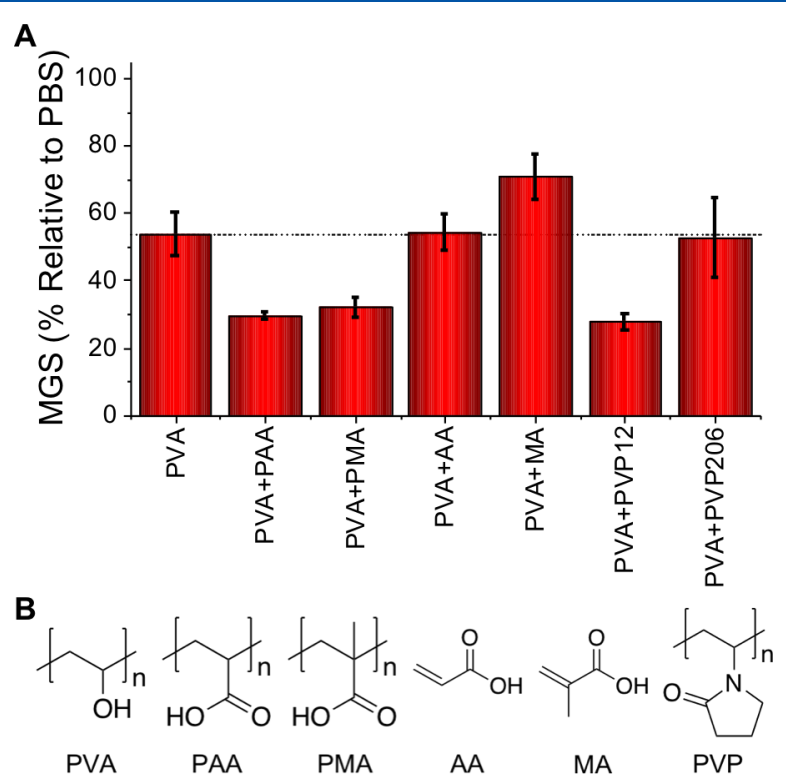

Figure 3. (A) IRI activity of $\mathrm{PVA}_{85}$ with indicated polymers added at $5 \mathrm{mg} \cdot \mathrm{mL}^{-1}$ compared to combinations of PVA and other polymers or monomers. The horizontal line at 53\% activity corresponds to the IRI of PVA alone. (B) Structures of depletants screened.

with addition of poly(acrylic acid), poly(methacrylic acid), and poly(vinyl pyrrolidone). In all cases the IRI activity was rescued, showing that these synergistic enhancements are not limited to PEG. These observations are particularly interesting as it has been shown that PVA/PEG mixtures are potent protein and bacterial cryopreservation agents, and this depletion-induced enhancement might be a factor. ${ }^{35,36}$ Control experiments were conducted using acrylic acid and methacrylic acid (monomers) and there was no IRI enhancement. Interestingly, high molecular weight PVP did not show activity. In the depletion model (Figure 1B), if the MW of the depletant is similar to the colloid, activity is lost, which these results would support. DLS analysis also supported that PVP 206 aggregates (Supporting Information).

It is important to highlight that the ammonium salt of poly(acrylic acid) can enhance the thermal hysteresis gap (another measure of antifreeze protein activity) of Type I antifreeze proteins and PVA. This was hypothesized to be due to specific basal face binding by poly(acrylic acid) and, in these reports, was only undertaken at saturation concentrations of the PVA. ${ }^{37,38}$

Our hypothesis for this activity enhancement relies on PVA being in a colloidal state (as proved by DLS) to enable the depletion effect to be valid. To provide evidence of our mechanism, two ice binding proteins ${ }^{23,24}$ were explored. AFP Type III was produced by recombinant expression in E. coli and AFGP8 (antifreeze glycoprotein from Antarctic Toothfish). As with PVA, the concentration of protein required to give $\sim 50 \%$ MGS was determined, and the impact of supplementing with PEG4k was evaluated, Figure 4. There was little or no enhancement in either case, in contrast to PVA. DLS of the AFPIII confirmed that it was not aggregating in up to $2 \mathrm{M} \mathrm{NaCl}$, and hence, no depletion effect is possible, as colloids of the IRI active material are required. 


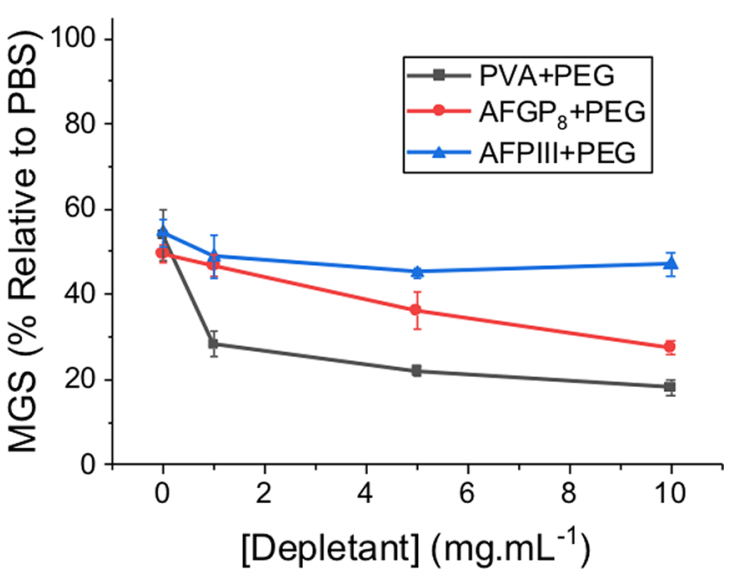

Figure 4. IRI activity with addition of PEG 4k: $[\mathrm{PVA}]=0.025 \mathrm{mg}$. $\mathrm{mL}^{-1},\left[\mathrm{AFGP}_{8}\right]=0.00008 \mathrm{mg} \cdot \mathrm{mL}^{-1},[$ AFPIII $]=0.00015 \mathrm{mg} \cdot \mathrm{mL}^{-1}$.

In conclusion, we have introduced a simple approach to rescue the ice recrystallization inhibition activity of PVA by exploiting colloidal depletion forces. Due to the high saline concentration between frozen ice grains, PVA aggregates to form colloids. Theoretical calculations showed that polymeric depletants can provide a driving force to deplete PVA colloids from solution and onto the ice. Experiments support this, showing that addition of polymers with no ice recrystallization inhibition activity enhanced the apparent activity of IRI, apparently due to the depletion effect. In line with theory, small molecule additives had no impact on the IRI activity and antifreeze proteins (which did not aggregate in saline) could not be enhanced due to the lack of colloids. These results are significant, as they present an accessible route to enhance the activity of synthetic IRI agents through simple addition of lowcost, water-soluble polymers.

\section{ASSOCIATED CONTENT}

\section{S Supporting Information}

The Supporting Information is available free of charge on the ACS Publications website at DOI: 10.1021/acsmacrolett.9b00386.

Full experimental details (PDF)

\section{AUTHOR INFORMATION}

\section{Corresponding Author}

*E-mail: m.i.gibson@warwick.ac.uk.

\section{ORCID}

Gabriele C. Sosso: 0000-0002-6156-7399

Matthew I. Gibson: 0000-0002-8297-1278

\section{Author Contributions}

The manuscript was written through contributions of all authors. All authors have given approval to the final version of the manuscript.

\section{Notes}

The authors declare no competing financial interest.

\section{ACKNOWLEDGMENTS}

M.I.G. holds an ERC Starting Grant (638661) and PoC Grant (789182). The Warwick Polymer RTP is acknowledged for SEC analysis. We thank A. L. DeVries (U. Illinois at Urbana Champagne, U.S.A.) for providing the AFGP8 and Prof P. Davies (Queens University, Canada) for providing the plasmids encoding AFP III. JSR Corporation is thanked for supporting this research. The research data supporting this publication can be found at http://wrap.warwick.ac.uk.

\section{REFERENCES}

(1) Davies, P. L. Ice-Binding Proteins: A Remarkable Diversity of Structures for Stopping and Starting Ice Growth. Trends Biochem. Sci. 2014, 39 (11), 548-555.

(2) Biggs, C. I.; Bailey, T. L.; Graham, B.; Stubbs, C.; Fayter, A.; Gibson, M. I. Polymer Mimics of Biomacromolecular Antifreezes. Nat. Commun. 2017, 8 (1), 1546.

(3) Voets, I. K. From Ice-Binding Proteins to Bio-Inspired Antifreeze Materials. Soft Matter 2017, 13 (28), 4808-4823.

(4) He, Z.; Liu, K.; Wang, J. Bioinspired Materials for Controlling Ice Nucleation, Growth, and Recrystallization. Acc. Chem. Res. 2018, 51 (5), 1082-1091.

(5) Biggs, C. I.; Stubbs, C.; Graham, B.; Fayter, A. E. R.; Hasan, M.; Gibson, M. I. Mimicking the Ice Recrystallization Activity of Biological Antifreezes. When Is a New Polymer "Active"? Macromol. Biosci. 2019, 1900082.

(6) Eniade, A.; Purushotham, M.; Ben, R. N.; Wang, J. B.; Horwath, K. A Serendipitous Discovery of Antifreeze Protein-Specific Activity in C-Linked Antifreeze Glycoprotein Analogs. Cell Biochem. Biophys. 2003, 38 (2), 115-124.

(7) Leclère, M.; Kwok, B. K.; Wu, L. K.; Allan, D. S.; Ben, R. N. CLinked Antifreeze Glycoprotein (C-AFGP) Analogues as Novel Cryoprotectants. Bioconjugate Chem. 2011, 22 (9), 1804-1810.

(8) Rajan, R.; Hayashi, F.; Nagashima, T.; Matsumura, K. Toward a Molecular Understanding of the Mechanism of Cryopreservation by Polyampholytes: Cell Membrane Interactions and Hydrophobicity. Biomacromolecules 2016, 17, 1882-1893.

(9) Stubbs, C.; Lipecki, J.; Gibson, M. I. Regioregular Alternating Polyampholytes Have Enhanced Biomimetic Ice Recrystallization Activity Compared to Random Copolymers and the Role of Side Chain versus Main Chain Hydrophobicity. Biomacromolecules 2017, 18 (1), 295-302.

(10) Mitchell, D. E.; Cameron, N. R.; Gibson, M. I. Rational, yet Simple, Design and Synthesis of an Antifreeze-Protein Inspired Polymer for Cellular Cryopreservation. Chem. Commun. 2015, 51 (65), 12977-12980.

(11) Drori, R.; Li, C.; Hu, C.; Raiteri, P.; Rohl, A.; Ward, M. D.; Kahr, B. A Supramolecular Ice Growth Inhibitor. J. Am. Chem. Soc. 2016, 138 (40), 13396-13401.

(12) Mitchell, D. E.; Clarkson, G.; Fox, D. J.; Vipond, R. A.; Scott, P.; Gibson, M. I. Antifreeze Protein Mimetic Metallohelices with Potent Ice Recrystallization Inhibition Activity. J. Am. Chem. Soc. 2017, 139 (29), 9835-9838.

(13) Graham, B.; Bailey, T. L.; Healey, J. R. J.; Marcellini, M.; Deville, S.; Gibson, M. I. Polyproline Is a Minimal Antifreeze Protein Mimetic and Enhances the Cryopreservation of Cell Monolayers. Angew. Chem., Int. Ed. 2017, 56, 15941-15944.

(14) Capicciotti, C. J.; Leclere, M.; Perras, F. A.; Bryce, D. L.; Paulin, H.; Harden, J.; Liu, Y.; Ben, R. N. Potent Inhibition of Ice Recrystallization by Low Molecular Weight Carbohydrate-Based Surfactants and Hydrogelators. Chem. Sci. 2012, 3 (5), 1408-1416.

(15) Inada, T.; Lu, S. S. Inhibition of Recrystallization of Ice Grains by Adsorption of Poly(Vinyl Alcohol) onto Ice Surfaces. Cryst. Growth Des. 2003, 3 (5), 747-752.

(16) Congdon, T.; Notman, R.; Gibson, M. I. Antifreeze (Glyco)Protein Mimetic Behavior of Poly(Vinyl Alcohol): Detailed Structure Ice Recrystallization Inhibition Activity Study. Biomacromolecules 2013, 14 (5), 1578-1586.

(17) Vail, N. S.; Stubbs, C.; Biggs, C. I.; Gibson, M. I. Ultralow Dispersity Poly(Vinyl Alcohol) Reveals Significant Dispersity Effects on Ice Recrystallization Inhibition Activity. ACS Macro Lett. 2017, 6 (9), 1001-1004.

(18) Burkey, A. A.; Riley, C. L.; Wang, L. K.; Hatridge, T. A.; Lynd, N. A. Understanding Poly(Vinyl Alcohol)-Mediated Ice Recrystalliza- 
tion Inhibition through Ice Adsorption Measurement and PH Effects. Biomacromolecules 2018, 19 (1), 248-255.

(19) Wowk, B.; Leitl, E.; Rasch, C. M.; Mesbah-Karimi, N.; Harris, S. B.; Fahy, G. M. Vitrification Enhancement by Synthetic Ice Blocking Agents. Cryobiology 2000, 40 (3), 228-236.

(20) Deller, R. C.; Vatish, M.; Mitchell, D. A.; Gibson, M. I. Synthetic Polymers Enable Non-Vitreous Cellular Cryopreservation by Reducing Ice Crystal Growth during Thawing. Nat. Commun. 2014, 5, 3244.

(21) Naullage, P. M.; Lupi, L.; Molinero, V. Molecular Recognition of Ice by Fully Flexible Molecules. J. Phys. Chem. C 2017, 121 (48), 26949-26957.

(22) Budke, C.; Koop, T. Ice Recrystallization Inhibition and Molecular Recognition of Ice Faces by Poly(Vinyl Alcohol). ChemPhysChem 2006, 7 (12), 2601-2606.

(23) Meister, K.; DeVries, A. L.; Bakker, H. J.; Drori, R. Antifreeze Glycoproteins Bind Irreversibly to Ice. J. Am. Chem. Soc. 2018, 140 (30), 9365-9368.

(24) Celik, Y.; Drori, R.; Pertaya-Braun, N.; Altan, A.; Barton, T.; Bar-Dolev, M.; Groisman, A.; Davies, P. L.; Braslavsky, I. Microfluidic Experiments Reveal That Antifreeze Proteins Bound to Ice Crystals Suffice to Prevent Their Growth. Proc. Natl. Acad. Sci. U. S. A. 2013, 110 (4), 1309-1314.

(25) Tanaka, S.; Ataka, M. Protein Crystallization Induced by Polyethylene Glycol: A Model Study Using Apoferritin. J. Chem. Phys. 2002, 117 (7), 3504-3510.

(26) Hudoba, M. W.; Luo, Y.; Zacharias, A.; Poirier, M. G.; Castro, C. E. Dynamic DNA Origami Device for Measuring Compressive Depletion Forces. ACS Nano 2017, 11 (7), 6566-6573.

(27) Yang, T.; Lei, Z.; Yang, S.; Chen, E.-Q. Depletion Driven SelfAssembly of Block Copolymer Solutions by Homopolymers. Phys. Chem. Chem. Phys. 2019, 21 (4), 2121-2127.

(28) Watanabe, K.; Tajima, Y.; Shimura, T.; Ishii, H.; Nagao, D. Depletion-Interaction-Driven Assembly of Golf Ball-like Particles for Development of Colloidal Macromolecules. J. Colloid Interface Sci. 2019, 534, 81-87.

(29) Stenger, P. C.; Isbell, S. G.; Zasadzinski, J. A. Molecular Weight Dependence of the Depletion Attraction and Its Effects on the Competitive Adsorption of Lung Surfactant. Biochim. Biophys. Acta, Biomembr. 2008, 1778 (10), 2032-2040.

(30) Kuhl, T.; Guo, Y.; Alderfer, J. L.; Berman, A. D.; Leckband, D.; Israelachvili, J.; Hui, S. W. Direct Measurement of Polyethylene Glycol Induced Depletion Attraction between Lipid Bilayers. Langmuir 1996, 12 (12), 3003-3014.

(31) Cheung, M. S.; Klimov, D.; Thirumalai, D. Molecular Crowding Enhances Native State Stability and Refolding Rates of Globular Proteins. Proc. Natl. Acad. Sci. U. S. A. 2005, 102 (13), 4753-4758.

(32) Ellis, R. J. Macromolecular Crowding: Obvious but Underappreciated. Trends Biochem. Sci. 2001, 26 (10), 597-604.

(33) Marenduzzo, D.; Finan, K.; Cook, P. R. The Depletion Attraction: An Underappreciated Force Driving Cellular Organization. J. Cell Biol. 2006, 175 (5), 681-686.

(34) Knight, C. A.; Wen, D.; Laursen, R. A. Nonequilibrium Antifreeze Peptides and the Recrystallization of Ice. Cryobiology 1995, 32 (1), 23-34.

(35) Mitchell, D. E.; Fayter, A. E. R.; Deller, R. C.; Hasan, M.; Gutierrez-Marcos, J.; Gibson, M. I. Ice-Recrystallization Inhibiting Polymers Protect Proteins against Freeze-Stress and Enable GlycerolFree Cryostorage. Mater. Horiz. 2019, 6, 364.

(36) Hasan, M.; Fayter, A. E. R.; Gibson, M. I. Ice Recrystallization Inhibiting Polymers Enable Glycerol-Free Cryopreservation of Microorganisms. Biomacromolecules 2018, 19 (8), 3371-3376.

(37) Funakoshi, K.; Inada, T.; Kawabata, H.; Tomita, T. Cooperative Function of Ammonium Polyacrylate with Antifreeze Protein Type I. Biomacromolecules 2008, 9 (11), 3150-3156.

(38) Funakoshi, K.; Inada, T.; Tomita, T.; Kawahara, H.; Miyata, T. Thermal Hysteresis Induced by Ammonium Polyacrylate as Antifreeze Polymer. J. Cryst. Growth 2008, 310 (14), 3342-3347. 